\title{
Prevalência de depressão em servidores do Instituto Nacional de Seguridade Social*
}

\author{
Leandro Aparecido Fonseca Missiatto ${ }^{1}$ \\ (D) https://orcid.org/0000-0002-6532-735X \\ Fabio Biasotto Feitosa ${ }^{2}$ \\ (D) https://orcid.org/0000-0001-6440-4993 \\ Tomás Daniel Menéndez Rodríguez ${ }^{2}$ \\ (D) https://orcid.org/0000-0003-3331-6346 \\ Maria Alice Simões de Mathis ${ }^{3}$ \\ (D) https://orcid.org/0000-0001-9552-0313
}

A depressão é o transtorno mental que mais cresce em incidência no mundo, gerando impacto de diversas ordens nas políticas de saúde coletiva dos países. Este estudo trata de uma pesquisa realizada com 132 servidores públicos do Instituto Nacional de Seguridade Social do estado de Rondônia cujo objetivo foi verificar a prevalência de depressão nessa amostra. Utilizou-se como instrumentos um questionário socioeconômico, elaborado pelos pesquisadores, e o Patient Health Questionnaire-9. Os resultados demonstram que a prevalência de depressão nesse grupo foi maior nos servidores do interior do estado, com alta escolarização e que permanecem estudando. Essas informações podem contribuir com a elaboração de específicas políticas de saúde mental para o contexto dos servidores do INSS/RO.

Descritores: Depressão; Prevalência; Servidores Públicos.

\section{Como citar este artigo}

Missiatto LAF, Feitosa FB, Rodríguez TDM, Mathis MAS. Prevalence of depression in servers of the National Institute of Social Security. SMAD, Rev Eletrônica Saúde Mental Álcool Drog. 2019;15(3):1-6. doi: https://dx.doi.org/10.11606/issn.1806-6976.smad.2019.000423 


\title{
Prevalence of depression in servers of the National Institute of Social Security
}

\begin{abstract}
Depression is the fastest growing mental disorder in the world, and has an impact on the collective health policies of the countries. This study deals with a survey conducted with 132 government employee of the National Institute of Social Security of the State of Rondônia, whose objective was to verify the prevalence of depression in this sample. A socioeconomic questionnaire developed by the researchers and the Patient Health Questionnaire-9 was used as instruments. The results show that the prevalence of depression in this group was higher in the government employee of the interior of the State, with high schooling and who are still studying. This information can contribute to the elaboration of specific mental health policies for the context of the INSS / RO employees.
\end{abstract}

Descriptors: Depression; Prevalence; Government Employee.

\section{Prevalencia de depresión en servidores del Instituto Nacional de Seguridad Social}

\begin{abstract}
La depresión es el trastorno mental que más crece en incidencia en el mundo, generando impacto de diversas órdenes en las políticas de salud colectiva de los países. Este estudio trata de una encuesta realizada con 132 funcionarios del Instituto Nacional de Seguridad Social del Estado de Rondônia cuyo objetivo fue verificar la prevalencia de depresión en esta muestra. Se utilizó como instrumentos un cuestionario socioeconómico, elaborado por los investigadores y el Patient Health Questionnaire-9. Los resultados demuestran que la prevalencia de depresión en este grupo fue mayor en los servidores del interior del Estado, con alta escolarización y que permanecen estudiando. Esta información puede contribuir a la elaboración de políticas de salud mental, específicas en el contexto de los servidores de INSS/RO.
\end{abstract}

Descriptores: Depresión; Prevalencia; Servidores Públicos. 


\section{Introdução}

A depressão é um problema de saúde pública mundialmente conhecido e se caracteriza por sintomas como sentimento de tristeza, falta de confiança, opiniões negativas de si, de outros e do futuro, perda de interesse em atividades e perturbação do sono e apetite $^{(1)}$. Estima-se ${ }^{(2)}$ que até o final da presente década a depressão será a segunda causa de incapacidade em saúde no mundo. Desse modo, a expansão da depressão tende, de um modo geral, a afetar as economias, setores produtivos e estratégias de saúde dos países.

No Brasil(3), a prevalência de autorrelato de diagnóstico de depressão em adultos, em 2013, foi de $7,6 \%$ (IC95\% 7,2 - 8,1), sendo maior em mulheres $(10,9 \%$; IC95\% 10,3 - 11,6) e em pessoas entre 60 e 64 anos (11,1\%; IC95\% 9,1 - 13,1); com ensino superior completo, 8,7\% (IC95\% 7,5 - 9,9); e para aqueles que se autodeclararam brancos (9,0\%; IC95\% 8,3-9,6).

Inúmeros trabalhos científicos apontam a prevalência da depressão em mulheres ${ }^{(4-7)}$ como importante característica do transtorno. Outra peculiaridade da depressão ressaltada por esses estudos é que a idade é uma variável considerável, já que com o aumento do tempo de vida também se eleva a taxa de prevalência de depressão em mulheres ${ }^{(3,8)}$. Essas pesquisas trabalham com a hipótese de que a prevalência superior desse transtorno em mulheres esteja associada às questões culturais que favorecem a busca feminina por auxílio médico, ampliando a margem de diagnósticos em relação ao universo masculino.

Além das características de gênero e idade, os estudos voltados à incidência da depressão comumente identificam a associação da variável escolarização com esse transtorno mental (9-10). Esses estudos sustentam a ideia de que fatores cognitivos, bem como aqueles promovidos pelos ambientes em que estão inseridas as pessoas com baixa escolarização, estão fortemente associados à etiologia da depressão.

No que concerne aos servidores públicos, não são muitos os trabalhos voltados à incidência da depressão, contudo os estudos que abordam a temática identificam a congruência das informações apontadas em pesquisas de base populacional(11).

Em Rondônia(12), a depressão foi o transtorno mental com maior prevalência entre servidores públicos federais no ano de 2011, acometendo consideravelmente o sexo feminino $(52,1 \%)$.

Nesse sentido, nosso estudo busca contribuir no entendimento das variáveis envolvidas na prevalência da depressão em servidores públicos, o que poderia fornecer subsídios para elaboração e execução de políticas de saúde adequadas para o contexto desses trabalhadores. Assim, o objetivo desta investigação foi verificar a prevalência de depressão em servidores públicos do Instituto Nacional de Seguridade Social no estado de Rondônia (INSS/RO).

\section{Método}

Esta pesquisa foi registrada e aprovada com o no. CAAE: 55655916.0.0000.5300 no Comitê de Ética em Pesquisa. Participaram 132 sujeitos servidores públicos no INSS de Rondônia, o que corresponde a 40,36\% do total de servidores da Instituição. Atualmente, a regional de Rondônia conta com 327 servidores, sendo 169 da capital e 158 do interior. A amostra foi composta por conveniência ( $61,4 \%$ da capital; $31,6 \%$ do interior). Seis cidades do interior participaram do estudo, sendo elas: Ariquemes, Ji-Paraná, Cacoal, Pimenta Bueno, Rolim de Moura e Vilhena. A escolha dessas cidades, além da capital, deu-se em virtude do tamanho das agências, número de beneficiários atendidos e posição geográfica no estado a fim de que pudessem garantir maior representatividade dos servidores do INSS de Rondônia.

Utilizou-se como instrumentos um questionário socioeconômico, elaborado pelos pesquisadores, e o Patient Health Questionnaire-9 (PHQ-9). O primeiro objetivou coletar dados referentes às condições socioeconômicas dos servidores do INSS. Consistiu em um questionário, autoaplicável, com perguntas que verificaram as seguintes características: sexo, idade, tempo de serviço no INSS, escolaridade e continuidade dos estudos.

Para avaliar a depressão, utilizou-se o (PHQ9). Esse instrumento avalia o Episódio de Depressão Maior conforme critérios diagnósticos do DSM-IV, em 9 questões $^{(10)}$. O instrumento tem a vantagem de ser autoaplicado, utilizando-se de uma escala Likert que avalia a frequência dos sintomas nas últimas duas semanas. Os indícios verificados pelo questionário são: humor deprimido, anedonia, problemas com o sono, cansaço físico, mudança no apetite ou peso, sentimento de culpa ou inutilidade, problemas de concentração, sentir-se lento ou inquieto e pensamentos suicidas. Há estudo que indica validade do $\mathrm{PHQ}-\mathrm{9}^{(13)}$, demonstrando que esse instrumento é adequado para rastreamento de episódio depressivo maior. O questionário classifica os níveis de depressão de acordo com a pontuação, sendo minimamente deprimido (0-4), pouco deprimido (5-9), moderadamente deprimido (10-14), muito deprimido (15-19) e severamente deprimido (20-27). Para este estudo, atribuiu-se a categoria não deprimido aos participantes que pontuaram zero.

A análise dos dados foi realizada de acordo com os critérios dos instrumentos aplicados e tratados por estatística descritiva e inferencial, utilizando-se o pacote SPSS para Windows versão 20.0. Considerando o tamanho da amostra e a característica dos testes, utilizou-se a análise de dados não paramétrica e teste 
de Mann-Whitney, adequados para pequenas amostras e sem distribuição homogêneas, garantindo, dessa forma, análises de significância e correlação de grupos independentes mais precisos ${ }^{(14)}$.

\section{Resultados}

A amostra da pesquisa foi composta por 39 pessoas do sexo masculino e 93 do sexo feminino, entre idades de 18 a 65 anos (mediana $=53$; média $=47,5$; desvio padrão $=13,31)$. Dos pesquisados, $38,6 \%$ possuíam ensino superior e $61,4 \%$ ensino médio. Permaneciam estudando um total de 35,6\%. A maior parte dos servidores ocupava cargo de nível médio $(82,6 \%)$, apenas $2,3 \%$ eram ocupantes de cargo de nível superior e $12,9 \%$ exerciam a função de gestor.

A Tabela 1 ilustra a prevalência total de manifestação de depressão em servidores das agências do INSS de Rondônia. Os dados apontam para um índice de $29,54 \%$ dos servidores com indicação de depressão moderada. O percentual de servidores com depressão muito acentuada ou severa foi de $18,18 \%$.

Tabela 1 - Frequência geral de depressão em servidores do INSS/RO/BR. Rondônia, Brasil, 2016

\begin{tabular}{lccccc}
\hline & $\begin{array}{c}\text { Não } \\
\text { depressivo }\end{array}$ & $\begin{array}{c}\text { Minimamente } \\
\text { deprimido }\end{array}$ & $\begin{array}{c}\text { Pouco } \\
\text { deprimido }\end{array}$ & $\begin{array}{c}\text { Moderadamente } \\
\text { deprimido }\end{array}$ & $\begin{array}{c}\text { Muito } \\
\text { deprimido }\end{array}$ \\
\hline N & 7 & 31 & 31 & 39 & $\begin{array}{c}\text { Severamente } \\
\text { deprimido }\end{array}$ \\
Média & - & 2,29 & 7,2 & 11,82 & 15 \\
Mediana & - & 2,00 & 7,00 & 12,00 & 16,73 \\
D.P. & - & 0,74 & 1,62 & 1,35 & 17,00 \\
\hline
\end{tabular}

Conforme demonstra a Tabela 2, a manifestação de depressão em servidores foi ligeiramente maior que a de servidoras, categorizando-os como moderadamente deprimidos, não havendo, contudo, diferença significativa por gênero.

Tabela 2 - Prevalência de depressão em servidores do INSS/RO/BR - 2016 por gênero. Rondônia, Brasil, 2016

\begin{tabular}{lcccc}
\hline & N & Média & D.P. & Significância \\
\hline Homens & 39 & 10,0 & 5,08 & 0,129 \\
Mulheres & 93 & 8,76 & 6,59 & 0,129 \\
\hline
\end{tabular}

A escolarização dos servidores do INSS se relaciona de forma positiva com a depressão (Tabela 3). Conforme dados, os servidores que possuem ensino superior e que continuam a estudar são mais depressivos que aqueles que possuem apenas ensino médio e não se encontram estudando.

Tabela 3 - Prevalência de depressão em servidores do INSS/RO/BR - 2016 por escolarização. Rondônia, Brasil, 2016

\begin{tabular}{|c|c|c|c|c|c|}
\hline & & $\mathrm{N}$ & Média & D.P. & Significância \\
\hline \multicolumn{2}{|c|}{ Ensino Médio } & 81 & 8,30 & 6,22 & $0,04^{*}$ \\
\hline \multicolumn{2}{|c|}{ Ensino Superior } & 51 & 10,45 & 5,98 & $0,04^{*}$ \\
\hline \multirow{2}{*}{$\begin{array}{l}\text { Continua } \\
\text { Estudando }\end{array}$} & Sim & 47 & 10,66 & 4,65 & 0,07 \\
\hline & Não & 85 & 8,28 & 6,78 & 0,07 \\
\hline
\end{tabular}

Dados PHQ-9. ${ }^{*} \mathrm{p}<0,05$.

Utilizando o coeficiente de correlação de Spearman rho, verificou-se não haver relação entre as variáveis idade e tempo de serviço com depressão (Tabela 4).
Tabela 4 - Relação de idade, tempo de serviço e depressão $(n=132)$ dos servidores do INSS/RO/BR 2016. Rondônia, Brasil, 2016

\begin{tabular}{lcc}
\hline & Idade & Tempo de serviço \\
\hline $\begin{array}{l}\text { Coeficiente de correlação } \\
\text { Spearman }\end{array}$ & $-0,31$ & $-0,45$ \\
Significância & 0,728 & 0,607 \\
\hline
\end{tabular}

Em se tratando de distribuição geográfica, houve significativa relação entre essa variável e a patologia depressiva. Servidores que trabalham nas cidades do interior são mais depressivos que os da capital (Tabela 5). Nota-se que o escore médio para depressão entre os servidores da capital foi de pouco deprimido, enquanto que no interior o nível foi de moderadamente deprimido.

Tabela 5 - Manifestação de depressão em servidores do INSS/RO/BR - 2016 por distribuição geográfica. Rondônia, Brasil, 2016

\begin{tabular}{lccccccc}
\hline & \multicolumn{3}{c}{ Capital } & \multicolumn{3}{c}{ Interior } & \multirow{2}{*}{ PHQ-9 } \\
\cline { 2 - 7 } & $\mathbf{N}$ & Média & D.P. & $\mathbf{N}$ & Média & D.P. & \\
\cline { 2 - 7 } & 81 & 8,10 & 5,9 & 51 & 10,76 & 6,4 & $0,03^{*}$ \\
\hline${ }_{*}<0,05$ & & & & & & &
\end{tabular}

\section{Discussão}

Entre 2005 e 2015, o número de casos de depressão cresceu cerca de $18 \%$ no mundo. No Brasil, a expectativa da depressão em 2015 foi de 5,8\% da população(5) 0 presente estudo identificou que mais de um quarto dos participantes da pesquisa apresentou depressão moderada, o que corresponde ao diagnóstico de Depressão Maior(15). Os resultados encontrados nesta pesquisa são maiores que os de outros estudos de base populacional( $7,9,11)$. Isso é possível graças à 
disponibilidade de vários instrumentos para identificação e mensuração da depressão, favorecendo a ligeira divergência entre os resultados. O PHQ-9, questionário utilizado neste estudo, por exemplo, possui um espectro com cinco níveis de identificação de depressão, indo de minimamente deprimido a severamente deprimido, o que possibilita o reconhecimento do transtorno em seus mais diversos estágios. Outro fator associado é a cultura, já que os grupos de servidores públicos diferem de regiões, natureza das funções e regime.

A taxa de prevalência de depressão nos servidores públicos do INSS/RO não difere significativamente de um sexo para outro. Esses dados não coadunam com os encontrados em outros estudos científicos. Conforme o relatório da Organização Mundial da Saúde sobre depressão e outras desordens mentais comuns ${ }^{(5)}$, a prevalência de depressão em mulheres é maior que em homens em todo o mundo, com variação de $2 \%$ entre os sexos.

Quanto à distinção da prevalência da depressão nos sexos, é importante considerar a consistência do instrumento utilizado para o viés de gênero. Nos Estados Unidos, um estudo com 5.995 participantes investigou se $0 \mathrm{PQH}-9$ é influenciado pela condição de gênero dos respondentes. A análise dos itens demonstrou ser desprezível o desempenho diferencial entre homens e mulheres, o que indica que o PHQ-9 não implica em prejuízo nas avaliações por diferenças de gêneros ${ }^{(16)}$. Sendo assim, as diferenças entre gêneros encontradas nesta pesquisa podem estar atreladas às características socioculturais do estado de Rondônia, e não ao instrumento utilizado. A literatura científica considera que os fatores culturais implicam em diferenças sociofamiliares desempenhadas por homens e mulheres, acarretando em consequências psicológicas significativas para perceber a depressão, bem como expressar os sentimentos depressivos ${ }^{(17)}$.

No que concerne à escolarização, a prevalência de depressão foi maior em servidores que mais estudaram e que continuam a estudar. Esses dados divergem de outras pesquisas que apontam que pessoas com menor escolarização sofrem mais por depressão(9-10). Todavia, há na literatura resultados parecidos com os encontrados neste estudo que identificaram correlação direta entre a maior escolarização e a prevalência de depressão(18). No Japão, país conhecido pela alta valorização dos estudos, quanto mais anos de estudos formais tem uma pessoa, mais suscetível à depressão se torna ${ }^{(5)}$. Diferenças como essas sugerem que quanto maior a escolaridade, mais consciência sobre si e sua saúde, fazendo com que aumentem as expectativas e cobranças sobre si mesmos, fatores que podem favorecer ao surgimento e desenvolvimento da depressão.

As variáveis tempo de serviço e idade não demonstraram relação significativa com a depressão dos servidores. No Brasil, pesquisas de base populacional que estudaram a relação entre idade e depressão apresentaram resultados divergentes ${ }^{(5,10-11,19)}$. Uma possibilidade é que os estudos de base populacional prestam informações mais genéricas, identificando de forma mais global a dinâmica da depressão, contudo este estudo trata de um público muito específico, que pelas suas características peculiares (regime de trabalho, metas, expectativas sociais e institucionais, vulnerabilidades às políticas governamentais etc.) podem orquestrar manifestação mais uniforme da depressão para idade e tempo de serviço.

Em se tratando das diferenças de prevalência em virtude da posição geográfica, observou-se que os servidores do INSS que estão lotados no interior do estado de Rondônia mostraram-se mais deprimidos que os da capital. No entanto, dados como esses são complexos, já que existem poucos inquéritos em nível estadual ou local para permitir comparação e os estudos disponíveis têm geralmente focado na prevalência da depressão em metrópoles e capitais(3,20-21).

Uma característica do interior de Rondônia, e possível interveniente para a preponderância de depressão nessas cidades, é o fato de terem sido predominantemente colonizadas por imigrantes do sul e sudeste do país, enquanto que Porto Velho recebeu forte influência nordestina, principalmente nos ciclos da borracha e construção da Estrada de Ferro Madeira Mamoré(22). Estudos demonstram que a prevalência de depressão é maior nas regiões Sul e Sudeste, e menor no Nordeste brasileiro. Esses elementos corroboram com a ideia de que a herança cultural esteja envolvida na expressão de depressão em cidades interioranas do estado de Rondônia(3). Outro fator implicado é a concentração de programas de saúde, coletivos e especializados, na capital, desfavorecendo o acesso à saúde nas cidades do interior.

Resta destacar que o estudo apresenta como limitação a pouca quantidade de trabalhos científicos que tenham estudado a prevalência de depressão em servidores do INSS/RO, o que dificultou a comparação com outros dados.

\section{Conclusão}

Este estudo verificou que os servidores do INSS/RO, lotados no interior do estado, com maior escolaridade e que continuam estudando são mais depressivos que os demais. A pesquisa revelou ainda que três em cada dez servidores da amostra estudada manifestaram Depressão Maior. As variáveis gênero, idade e tempo de serviço não mostraram possuir relação com a depressão. De modo geral, essas informações indicam que esse transtorno é comum no Instituto Nacional de Seguridade Social de Rondônia, o que sugere a implementação de específicas políticas de saúde mental para o enfrentamento da depressão nesse contexto de trabalho.

\section{Agradecimentos}

A Márcia Cristina Pinto, Diretora Executiva do INSS/ RO e Maria Auxiliadora, Assistente Social do INSS/RO, pela acolhida e apoio em toda fase de execução da pesquisa. 


\section{Referências}

1. Patel V, Lund C, Hatherill S, Plagerson S, Corrigall J, Funk $M$, et al. Mental disordens: equity and social determinants. In: World Health Organization (WHO). Equity, social determinants and public health programmes. [Internet]. 2010 [cited Mar 13 2017]. Available from: http://www.who.int/mediacentre/ factsheets/ fs369/en/.

2. World Health Organization. Equity, social determinants and public health programmes. [Internet]. 2010 [cited Mar 15 2017]. Available from: http://www.who.int/ sdhconference/resources/EquitySDandPH_eng.pdf.

3. Stopa SR, Malta DC, Oliveira MM, Lopes CR, Menezes PR, Kinoshita RT. Prevalência do autorrelato de depressão no Brasil: resultados da Pesquisa Nacional de Saúde, 2013. Rev Bras Epidemiol. 2015 Dez; 18(suppl 2):170-80.

4. Guilland R, Cruz RM. Prevalência de transtorno mental e comportamental em trabalhadores de indústrias de abate de suínos e aves no sul do Brasil. Rev Colombiana Psicol. 2017; 26(1):163-77.

5. World Health Organization (WHO). Depression: and other common mental disorders: global health estimates. [Internet]. 2017 [cited Mai 15 2017]. Available from: http://apps.who.int/iris/bitstream/10665/254610/1/ WHO-MSD-MER-2017.2-eng.pdf?ua=1.

6. Richards D, Sanabria, AS. Point-Prevalence of depression and associated risk factors. J Psychol. 2017, 148(3):305-26.

7. Boing AF, Melo GR, Boing AC, Moretti-Pires RO, Peres KG, Peres MA. Associação entre depressão e doenças crônicas: um estudo populacional. Rev Saúde Pública. jun 2012; 46(4):617-23.

8. Guerra M, Pima AM, Ferri CP, Acosta D, Gallardo S, Huang $Y$, et al. A comparative cross-cultural study of prevalence of late life depression in low and middle income countries. J Affective Disorders. 2016 Jan; 190:362-8.

9. Molina MRAC. Wiener CD, Branco JC, Jansen K, Souza LDM, Tomasi E, et al. Prevalência de depressão em usuários de unidades de atenção. Rev Psiq Clín. 2012; 39(6): 194-7.

10. Munhoz TN. Prevalência e fatores associados à depressão em adultos: um estudo de base populacional. [Dissertação]. Rio Grande do Sul (RS); Universidade Federal de Pelotas, Pelotas, 2012. 136 p.

11. Abdalla MACS. Prevalência de sintomas de depressão em servidores de uma universidade pública [Dissertação]. Juiz de Fora (MG): Universidade de Juiz de Fora; 2014. 88 p.
12. Schlindwein VLDC, Morais PR. Prevalência de transtornos mentais e comportamentais nas instituições públicas federais de Rondônia. Cad Psicol Soc Trabalho. 2014; 17(1):117-27.

13. Santos IS, Tavares BF, Munhoz TN, Almeida LSP, Silva TB, Tams BD, et al. Sensibilidade e especificidade da Patient Health Questionnaire-9 (PHQ-9) entre adultos da população geral. Cad Saúde Pública. 2013 Aug; 29(8): 1533-43.

14. Hackbarth AA Neto, Stein CE. Uma abordagem dos testes não-paramétricos com utilização do Excel. [Internet]. 2005[Acesso 13 jun 2016]. Disponível em: http://home.furb.br/efrain/matematica/minicurso/ artigo_11_09_2003.doc.

15. Bergerot CD, Laros JA, Araujo TCCF. Avaliação de ansiedade e depressão em pacientes oncológicos: comparação psicométrica. Psico-USF. 2014 Ago;19(2): 187-97.

16. Thibodeau MA, Asmundson GJ. The PHQ-9 assesses depression similarly in men and women from the general population. Personal Individual Differences. 2014; 56:149-53.

17. Justo LP, Calil HM. Depressão: o mesmo acometimento para homens e mulheres? Rev Psiquiatr Clín. 2006; 33(2): 74-9.

18. Gao YQ, Pan BC, Sun W, Wu H, Wang JN, Wang, L. Depressive symptoms among chinese nurses: prevalence and the associated factors. J Adv Nurs. 2012 Mai; 68(5): 1166-75. 19. Bromet E, Andrade LH, Hwang I, Sampson NA, Alonso J, Girolamo G, et al. Cross-nation epidemiology of DSM-IV major depressive episode. BMC Med. [Internet]. 2011 Jul. [cited Jan 20 2017]. Available from: https://bmcmedicine.biomedcentral.com/ articles/10.1186/1741-7015-9-90

20. Gonçalves DA, Mari JJ, Bower P, Gask L, Dowrick C, Tófoli LF, et al. Brazilian multicentre study of common mental disorders in primary care: rates and related social and demographic factors. Cad Saúde Pública. 2014 Mar; 30(3): 623-32.

21. Cunha RV, Bastos GAN, Duca GFD. Prevalência de depressão e fatores associados em comunidade de baixa renda de Porto Alegre, Rio Grande do Sul. Rev Bras Epidemiol. 2012; 15(2):346-54.

22. Souza VA. Rondônia, uma memória em disputa [Tese]. São Paulo (SP): Universidade Estadual Paulista; 2011. 202 p.

Recebido: 26.09.2017 Aceito: 31.07 .2018 